\title{
Measurement of Cosmic Ray Nuclei with GRAINE2015
} \author{
Yoshimoto $^{f}$ \\ ${ }^{a}$ Department of Fundamental Science, Okayama University of Science \\ Okayama 700-0005, Japan \\ ${ }^{b}$ Graduate School of Science, Okayama University of Science \\ Okayama 700-0005, Japan \\ ${ }^{c}$ Faculty of Human Development, Kobe University \\ Kobe-city 657-8501, Japan \\ ${ }^{d}$ Aichi University of Education \\ Kariya-city, 448-8542, Japan \\ e JAXA/ISAS \\ Sagamihara-city, 252-5210, Japan \\ ${ }^{f}$ Dept of Physics, Faculty of Science, Nagoya University \\ Nagoya 464-8602, Japan \\ E-mail: iyono@das.ous.ac.jp
}

Atsushi lyono ${ }^{* a}$, Saya Yamamoto ${ }^{b}$, Shigeki Aoki ${ }^{c}$, Toshio Hara $^{c}$, Kenji Kuretsubo ${ }^{c}$ Toshitsugu Marushima ${ }^{c}$, Haruka Matsumoto $^{c}$, Fukashi Mizutani $^{c}$, Keita Ozaki ${ }^{c}$, Emi Shibayama ${ }^{c}$, Atsumu Suzuki $^{c}$, Satoru Takahashi ${ }^{c}$, Yurie Tateishi ${ }^{c}$, Misato Yabu ${ }^{c}$, $K^{K y o h e i ~} \operatorname{Yamada}^{c}$, Koichi Kodama $^{d}$, Kaname Hamada $^{e}$, Hiroaki Kawahara ${ }^{f}$, Ryosuke $\operatorname{Komatani}^{f}$, Masahiro Komatsu $^{f}$, Motoaki Miyanishi $^{f}$, Misaki Morishita $^{f}$, Kunihiro $^{\prime}$ Morishima $^{f}$, Mitsuhiro Nakamura ${ }^{f}$, Naotaka Naganawa ${ }^{f}$, Toshiyuki Nanano ${ }^{f}$, Akira Nishio $^{f}$, Kimio Niwa $^{f}$, Naoto Otsuka ${ }^{f}$, Hiroki Rokujo ${ }^{f}$, Osamu Sato ${ }^{f}$, and Masahiro

GRAINE(Gamma Ray Astro-Imager with Nuclear Emulsion) experiment consists of a balloon borne emulsion chamber and emulsion film shifting system launched on May 6, 2015 in Australia and its acceptance and flight duration is $3780 \mathrm{~m}^{2}$ sr and 14 hours at $37 \mathrm{~km}$ above sea level, respectively. The main scientific goals of GRAINE2015 are the precise measurement of sub GeV gamma-rays from Vela pulsar, the measurement of charm particle production cross section at high altitude in the air and the study of cosmic ray nuclei compositions. The nuclear emulsion films used in GRAINE2015 will be able to measure cosmic ray nuclei from hydrogen nuclei to more than iron nuclei. The measurement of the chemical composition of cosmic ray nuclei in the MeV$\mathrm{TeV}$ energy range will reveal new insights about the cosmic ray nuclei life in the our galaxy, from their origin to the propagation in the interstellar medium. The Cosmic ray nuclei absolute charge $\mathrm{Z}$ is measured along the trajectory in the GRAINE2015 emulsion films by using high speed emulsion track selector (HTS). This allows to scan the entire emulsion film within the zenith angle less than 63 degree. The charge measurement methods in HTS scan data are here explained, and their performance presented.

35th International Cosmic Ray Conference - ICRC2017

10-20 July, 2017

Bexco, Busan, Korea

\footnotetext{
* Speaker.
} 


\section{Introduction}

The observation of astronomical gamma rays provides the origins and acceleration mechanism of high energy cosmic rays in the universe such as compact objects and the galaxies. Recent Fermi LAT satellite experiments [1 2] had reported the observation results on more than three thousand gamma ray point sources including 30 percent unidentified stellar objects. The more precise angular resolution has been needed in the gamma ray's energy range of a $10 \mathrm{MeV}-100 \mathrm{GeV}$ to explore the gamma ray production mechanism of hadronic origins and leptonic ones. Furthermore, precise measurement of the hydrogen flux in primary cosmic ray above $1 \mathrm{GV}$ rigidities were reported by the AMS collaboration [3] and the spectral index hardening at high rigidities were found.

Nuclear emulsion films are the precise tracking detectors which registers the charged particle trajectories with less than one micrometer accuracies in the three-dimensional space. To measure the arrival direction and energy of gamma rays in nuclear emulsion chambers, the leading point of the electron-positron pair created by gamma-ray conversion have been measured with submilliradian angular resolution in one emulsion film, the each electron's direction and its momentum can be precisely determined by tracking them.

The Japan-Australia JAXA collaborative balloon borne experiment (GRAINE 2015) [4] was successfully launched from the Alice Springs balloon launching station to Longreach with a 3780 $\mathrm{cm}^{2}$-aperture telescope for the 14.4 hours duration, including 11.5 hours of the level flight at the altitude of $36.0 \mathrm{~km}$ to $37.4 \mathrm{~km}\left(4.8 \mathrm{~g} / \mathrm{cm}^{2}\right.$ to $\left.3.9 \mathrm{~g} / \mathrm{cm}^{2}\right)$ in May 6,2015 . The primary goals of GRAIN2015 were to confirm the overall system performance including chemical processing and emulsion shifter technlogies and to observe the morphology of the well-known and brightest gamma-ray object such as the Vela pulsar with the gamma-ray emulsion telescope.

The nuclear emulsion films had been exposed to cosmic radiation at the $4.4 \mathrm{~g} / \mathrm{cm}^{2}$ atmospheric depth directly, and we expected comic ray nuclei components. Cosmic ray nuclei provide the information on the origin, propagation and acceleration mechanism of cosmic rays. In GRAINE 2015 balloon flight [4], the detections of these cosmic ray nuclei components were able to be examined with image analysis technologies as well as time-stamper systems.

\section{The structure of emulsion telescope system}

The emulsion chamber structure of the gamma-ray telescope [4 [5 which consists of a converter part, time-stamper part, calorimeter part, and an attitude monitor systems is shown in Figure 11 The materials of a converter part make the incident gamma rays convert into electron-positron pairs and these electrons have been registered as three dimensional tracks in the stacks of nuclear emulsion films. The time-stamper part which consists of triple emulsion film shifters like a hour, a minute and a second hand of an analog clock shown in Figure 2 provides a track arrival time (or time-stamp) information for all charged particles. After the recognition and reconstruction of electron pairs, the arrival time and direction as well as the momentum were determined by measuring the track deflection angle due to multiple Coulomb scattering. The arrival timing obtained by time-stamping of electron pairs is combined with three star camera images deployed at the balloon gondola structure shown in Figure 3 and their arrival directions were converted into the celestial 
coordinate. These cameras monitored the attitude of gondola to observe the stars positions. The trajectory of charged particles measured int the emulsion films enables to estimate their momentum by using multiple Coulomb scattering method up to several hundred MeV/c.

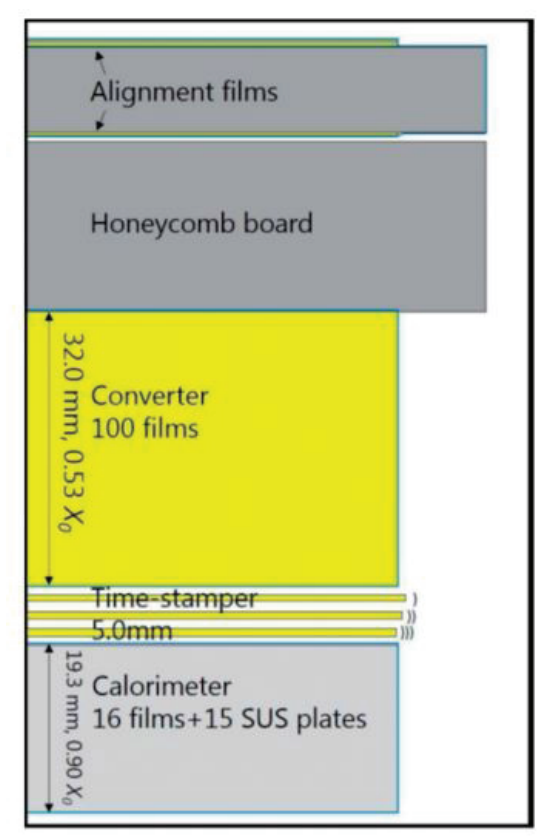

Figure 1: GRAINE 2015 emulsion chamber structure which consists alignment emulsion films, honeycomb spacer, converter part, time-stamper part and calorimeter part.

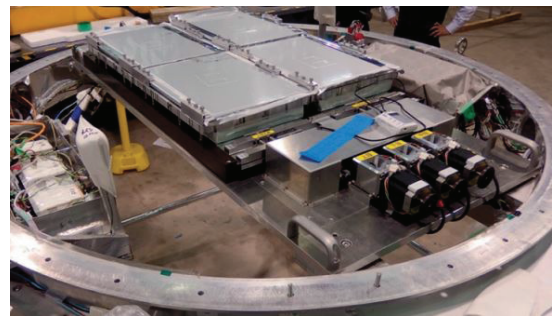

Figure 2: Emulsion chambers in the pressure vessel of the gondola. Three motorized shifters control emulsion film locations with GPS time. In the time-stamper part, the three different time period such as hour, minute and second hand of an analog clock worked.

\section{Emulsion film analysis}

After recovering the emulsion films at Longreach Australia May 6,2015, exposed emulsion films were sent to Sydney University in order to complete chemical developments as soon as possible because of background accumulations and minimizing the effect of fading of latent images. The further chemical treatment for these developed films had been handled at Kobe university. Typical thickness of emulsion part of films after developed was very thin (such as $\sim 40 \mu \mathrm{m}$ ). For the effective image recognition procedures, we need the original emulsion thickness when exposing them to cosmic radiations. The glycerin swelling process have been carried out for all emulsion films to swell the gelatin part of nuclear emulsion by glycerin infiltrating. The recovered thickness was achieved up to 90 percent of the nominal thickness.

In emulsion track recognition process, GRAINE has used high performance and high speed track selector HTS (Hyper Track Selector shown in Figure 44 developed and implemented at the Fundamental Particle Laboratory(F-lab.) 6, 7] in Nagoya university. HTS achieves over $5000 \mathrm{~cm}^{2}$ per hour scanning speed within the zenith angle less than 60 degree. The HTS provides the general scan approach for the emulsion analysis with wide zenith angle allowance for cosmic ray nuclei 

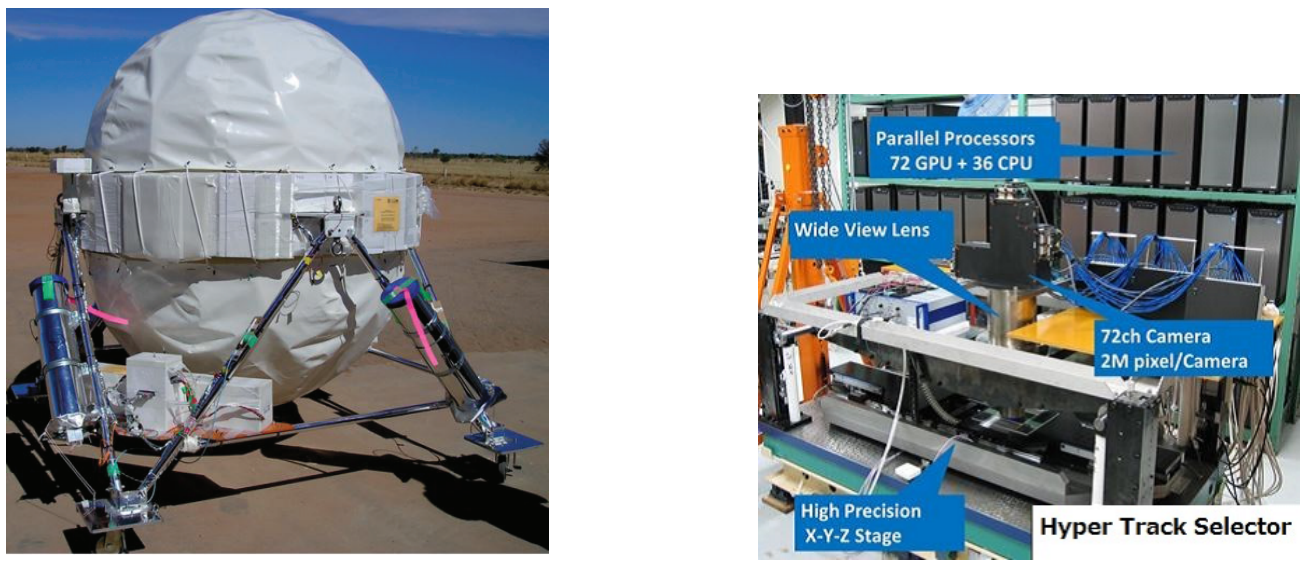

Figure 3: The GRAINE 2015 gondola with thermal Figure 4: Hyper Track Selector(HTS) implemented at shielding. The three star cameras are deployed at the Fundamental particle laboratory in Nagoya university. truss structure and each camera views the three differ-

ent directions of the sky.

studies, elemental particle physics as well as muon tomography technology. To recognize charged tracks in the emulsion films, HTS acquired sliced images in both upper and bottom emulsion layers by slicing them in to thirty three binary images in GPU memory space. The GPU pipeline logically shifted them in two dimensional directions and summed the image overlapping along the specified zenith angle and orientation angle. The Pulse Height $(\mathrm{PH})$ and Pulse Height Volume(PHV) were defined as the number of overlapped pixels and the total number of $\mathrm{PH}$ values above threshold values(typically 7 or 8), respectively. HTS scanning algorithm were shown in Figure 5 The nominal allowance of zenith angle has extended up to 63 degree $(\tan \theta \leq 2.0)$ in GRAINE2015 analysis and the track recognition efficiency became more than 95 percent.

\section{Ionization loss measurement with pulse height volume(PHV) analysis}

The pulse height volume defined in the section 3 could privide the ionization loss information of each track's $(Z / \beta)$. We selected about fifty charged tracks in both $\tan \theta<0.6$ and $\tan \theta>1.0$ of which PHV values are relatively high and obtained their raw images by using HTS manual mode operations shown in Figure 6 Most of them were associated with knock-on electrons $(\delta$ rays), which indicated the incidence of cosmic ray heavier nuclei. Because the number of $\delta$ rays were very limited, we did not come in detail for its relation to PHV data.

We analyzed about $10^{6}$ tracks in each emulsion film and followed them among one hundred emulsion film stacks. The tracking efficiencies between three films were typically 95 percent. We examined the PHV distribution in one emulsion film. The PHV data for single charged tracks depend on their incident zenith angles because all grains were overlapped in case of $\tan \theta=0$ and not overlapped in horizontal tracks. The PHV distributions for each zenith angle group were shown int Figure 7. The mode values of PHV distributions were decreasing as their zenith angles were increasing, due to the grain separation of single charged tracks. This depth of field for objective lens system cause the grain overlapping effect and makes relatively higher PHV values in case of 

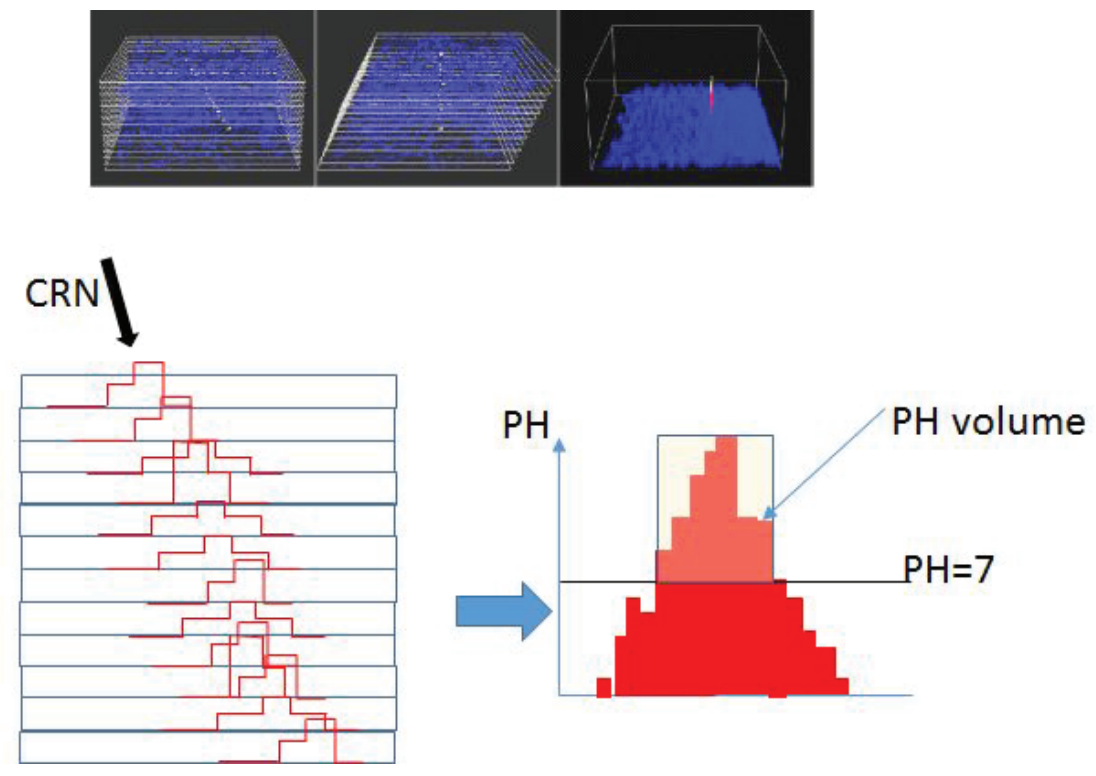

Figure 5: HTS algorithm to search for tracks and definitions. The symbol:CRN represents incident charged cosmic ray which penetrating emulsion layer and, $\mathrm{PH}$ and $\mathrm{PH}$ volume(PHV) are the number of overlapped pixels and summed pixel number above $\mathrm{PH}$ threshold greater than 7, respectively.

vertical tracks. To reduce this effect, the swelling processes for developed emulsion films are very effective as well as effective track recognitions. In image analysis procedures of HTS algorithm, image filtering along the focal direction have been took into account such as FIR/IIR type digital signal processing. On the other hand, the PHV highest values became larger as zenith angles are increasing, because the horizontal tracks blurred some multiple sliced images simultaneously. We demonstrated the normalized PHV distribution by fixing the mode value equal to 50, the second peak PHV value above 100 and minimum PHV values. In Figure 8, obtained nomalized PHV distributions for several zenith angle groups are shown. Around the PHV mode value, these distributions scattered over with zenith angles. This could come from the grain overlapping effects described above. But nomalized PHV distributions were well described with one shape above PHV $\geq 100$. This could provide some universality of the relation of PHV to zenith angles. In these distributions, we clearly obtained the second peak in each zenith angle group. The zenith angle dependency of nomalization parameters (minimum, mode and maximum PHV values) were plotted as function of zenith angles in Fugure 9. The charged particles are able to be clustered by normalized pulse height volumes(NPHV) and energy dependency of NPHV is not clearly seen yet.

\section{PHV distributions at both sea level and flight level}

GRAINE 2015 have developed and utilized emulsion film shifters in order to determine charged track arrival timing described above. This system enable us to distinguish the accumulation of background $\mu$-on tracks at sea level and cosmic rays in flight by using track arrival timing. We set up emulsion shifters at special coordinates such as an origin when waiting for the balloon launch. In the flight, three shifter stages moved in each proper designed period. The PHV distribution of the tracks which penetrated the chambers at sea level was shown in Figure 10, The horizontal axis 


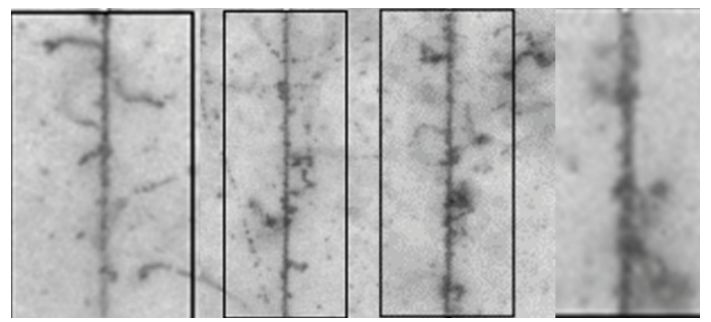

Figure 6: Example raw images for relatively higher PHV tracks. Images are processed by applying dark peak fold filter and accumulated along the track directions.
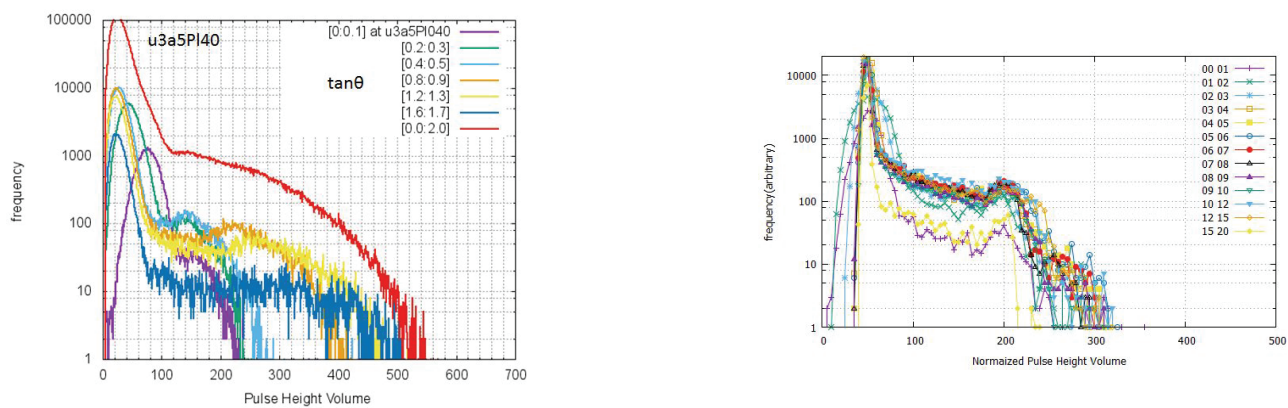

Figure 8: The normalized PHV distribution at emul-

Figure 7: PHV distribution at one emulsion layer. sion layer. Horizontal axis represents the normalized Horizontal axis represents PHV. Each line color rep- PHV. Each color represents zenith angle $(\times 10)$ group. resents zenith angle grouping.

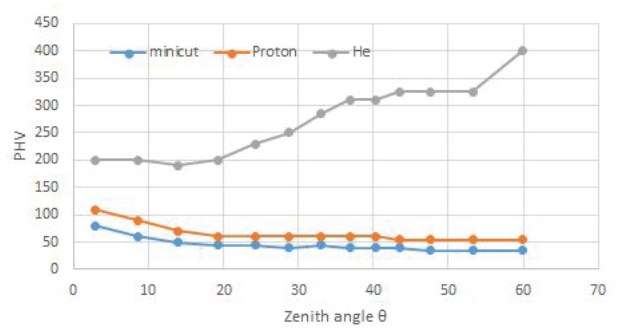

Figure 9: The dependence of normalized PHV parameters of track zenith angles. The minimum, mode and maximum PHV values are indicated by minimum ionized track, proton and helium respectively.

represents the PHV summation between 4 emulsion layers, and then PHV values were twice as large as the previous ones. In this distribution, the mode PHV $(\sim 180)$ mainly came from single charged particles such as atmospheric $\mu$-on. At PHV $=300$, the second peak of PHV distribution was unseen and the maximum PHV does not reach 500. On the other hand, the PHV distribution obtained in the balloon flight shown in Figure 11 differed from the previous one especially above $P H V=300$. We have clearly seen the second peak at around PHV=400 and the maximum values extends to $\mathrm{PHV}=600$. This difference came from cosmic ray Helium and heavier nuclei contributions in the high PHV range. And this result proved the potential of cosmic ray nuclei measurement in GRAINE projects. 


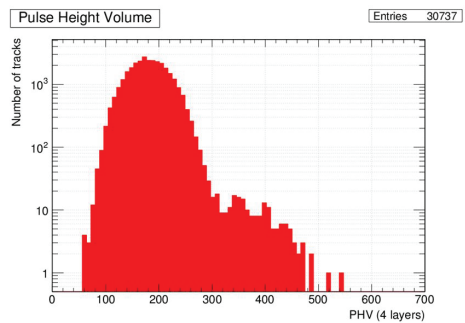

Figure 10: PHV distributions at ground level before launching the balloon.

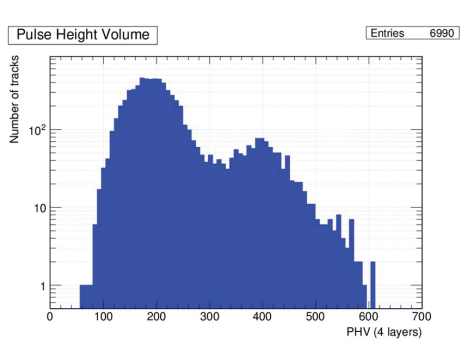

Figure 11: PHV distributions in the balloon flight

\section{Trajectory reconstruction by using nearby tracks}

To determine ionization loss signals from PHV, we have to determine the velocity beta of each track. In the GRAINE chamber, we use multiple Coulomb scattering angles of charged tracks. The charged tracks are realigned by using their nearby tracks with the weight of their energies. The alignment parameters between two emulsion plates are assumed as four affine parameters, Cartesian shift values and the depth of another layer from the one layer. We applied LevenbergMarquardt method [8] for the optimization procedures and track linearities are used as weight of data quality. To avoid low energy track contamination when calculating these quantities, we made the criteria of two sigma displacement cut, and also to eliminate large scale emulsion plate distortion. We selected three thousand nearby tracks as one group for optimization calculations. Typical reconstruction accuracies are less than 2 micrometer as shown inf Figure12 Because track connection quality depends on its momentum, normalized pulse height volume described above are plotted as function of track displacement $\sqrt{d x^{2}+d y^{2}}$ as shown in Figure 13
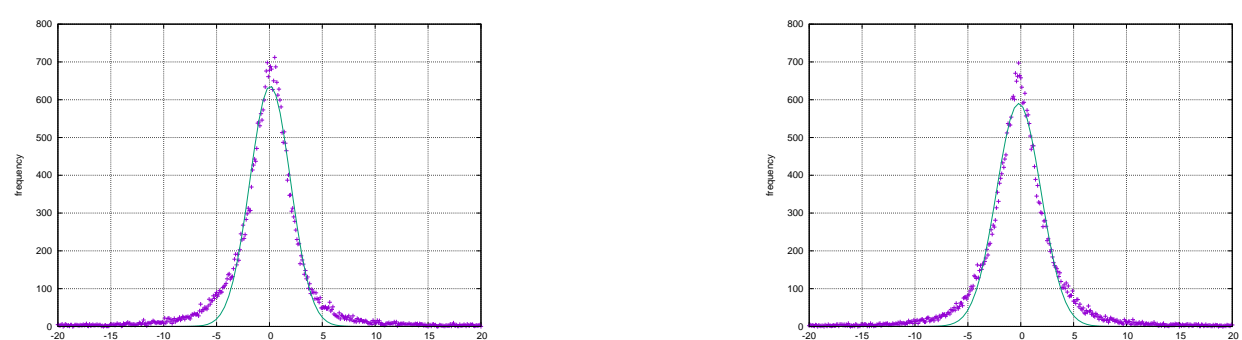

Figure 12: (dx,dy) distribution obtained by Levenberg-Marquardt method for alignment calculation

\section{Conclusion}

The GRAINE projects successfully completed the balloon flight at Alice Springs, Australia for 14 hours duration in 2015. The overall system worked well within the expected accuracies. The nuclear emulsion film handling before and after the balloon flight at both Australia and Japan was well controlled. 


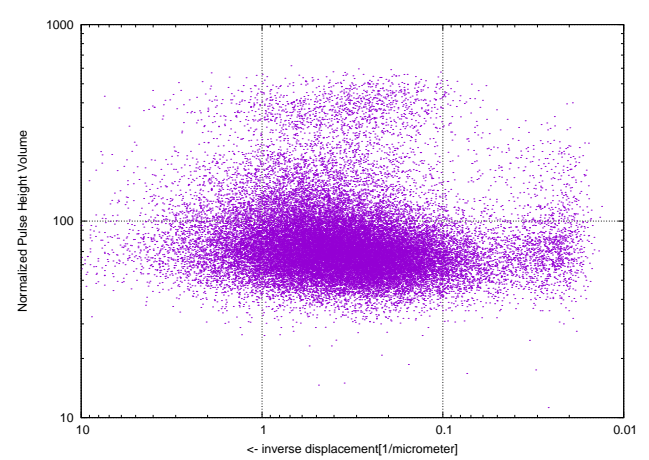

Figure 13: Scatter plot of normalized pulse height volume as function of track displacements

The pulse height volume obtained by HTS track recognition process were able to be used for cosmic ray nuclei identification. The PHV distributions provided cosmic ray nuclei composition information. Finally, the time-stamper system can distinguish the ground level EAS muons and flight-components of direct cosmic rays, by using the arrival timing of cosmic rays.

The qualitative characteristics of pulse height volume ( PHV ) were successfully derived from this analyses. We have to understand the quantitative estimation of PHV dependencies of incident zenith angles.

\section{Acknowledgments}

This work was partially carried out by the joint usage / research program of the Institute of Materials and Systems for Sustainability (IMaSS), Nagoya University.

\section{References}

[1] C. GENE et al., FERMI-LAT DETECTION OF PULSED, Astrophys. J. L.,L13,2014, 797

[2] F. ACERO et al., FERMI LARGE AREA TELESCOPE THIRD, Astrophys. J., 218, 2015,23

[3] M. Aguilar et al., Precise Measurement of the Proton, Phys. Rev. Lett. ,114,2015, 171103

[4] S. Takahashi et al., GRAINE 2015, a balloon-borne emulsion , Prog. Theor. Exp. Phys., 073F01,2016, 12

[5] H. Rokujo et al., Development of a balloon-style pressure vessel for, Proc. 34th Int. Cosmic Ray Conference The Hague, PoS(ICRC2015),2015,1021

[6] K. Niwa et al., Auto scanning and measuring system for the emulsion chamber, Proc. Int. Cosmic Ray Symp. High Energy Phenomena Tokyo,1974, 149.

[7] T. Nakano, The Newest Technique for Nuclear Emulsion Readout, J. of The Society of Photographic Science and Technology of Japan ,79,1,2015, 54-59

[8] M. Galassi et al, GNU Scientific Library Reference Manual (3rd Ed.), ISBN 0954612078,2009 